\title{
Study on Community Environment Assessment of Urban Reconstruction in Wenchuan Earthquake Stricken Areas-Take Dujiangyan One Neighborhood as an example
}

\author{
Sun Xian \\ College of Architecture and Urban Planning, Tongji University, No. 1239, Siping Road, Shanghai, \\ China
}

384271261@qq.com

\begin{abstract}
Key words: Wenchuan earthquake disaster area; reconstruction; community environment; evaluation
\end{abstract}

\begin{abstract}
Taking the evaluation of urban environmental reconstruction in urban areas in Wenchuan earthquake as an objective. this paper constructs 6 criterion layers of "location scale", "space environment", "facilities support", "security guarantee", "community management" and "community atmosphere" . Taking Dujiangyan One Neighborhood as an example, this paper empirically studies the weight calculation process of AHP and fuzzy comprehensive evaluation. The sub-index evaluation and comprehensive evaluation analysis of the sample are presented, and the feedback information is put forward.
\end{abstract}

\section{Introduction}

The 5.12 Wenchuan earthquake brought heavy losses to the disaster-hit areas, destroyed a large number of houses and displaced people. With the leadership and support of the party and the state, the post-Wenchuan earthquake reconstruction rebuilt itself in a very short period of time to make the disaster-stricken areas look new and the victims have gradually taken the normal life track. In post-disaster reconstruction, the reconstruction of community environment is an important manifestation of the reconstruction of environment. It is the basic guarantee for the reconstruction of environment and the ability of residents in disaster-stricken areas to resume normal life, and relates to the healthy and sustainable long-term development of disaster-stricken areas.

Community is a microcosm of the macro society, people's feelings of community construction is the most direct, is the most familiar and concerned. As the basic unit of urban composition, community reconstruction is an important part of post-disaster reconstruction and an important cornerstone of the success of post-disaster reconstruction. Based on the reconstruction of community environment, this paper tests the effectiveness of post-disaster reconstruction within a certain range through community environmental assessment, and provides feedback suggestions and theoretical reference for the long-term development of disaster-stricken areas.

\section{Research Category}

In urban planning, community more refers to concepts such as city and living, which is the sum of the people living in a particular area of the city and the space in which they live. Some people more emphasis on the characteristics of space, geographical scope, the community as a settlement of a certain level. Such as families, neighborhoods, communities, towns, cities, regions and other small to large series of one level.

This paper argues that community refers to a social group formed by design and organization in a specific region, has a common interest relationship and social interaction, and is a humanities and space complex unit in the city. 


\section{Evaluation System Construction}

Evaluation system construction principles

(1) Concise Scientific Principles. The design of index system must be based on science, reflecting objectively and truthfully the composition of environmental performance targets of the community, and reflecting the dominance of environmental target indicators. Moreover, the index system should not be too thin and too narrow.

(2) Systematic Principle. The factors influencing the community environment are varied, so building the index system of community environmental assessment is a complicated systematic project. To really reflect the basic characteristics of the living environment, we must take into account all aspects of the quality of living environment. Therefore, in building each set of indicators for each aspect, the indicators should be independent and interrelated. From every aspect of the macro to the micro every indicator must have a certain level, the index system must be hierarchical, so as to form a complete evaluation system. Only such a rating system can reflect the comprehensive environmental quality of living environment and the quality of the environment in all aspects of each category.

(3) Measurable Principle. To grade and judge the quality of living environment, it is required to quantify the quality of living environment. The quantitative principle is: the evaluation of indicators can be quantified directly or can be indirectly assigned to quantify for qualitative indicators.

(4) Unity Principle. The principle of unity is to unify the meaning of the evaluation index, the scope of application. Only the indicators under the principle of uniformity have the comparability of evaluation results.

(5) Feasibility Principle. The principle of feasibility is that the quality of the evaluation index of living environment should be easy to obtain. Because some indicators can comprehensively and clearly reflect the quality of the community environment, it takes a lot of manpower, financial resources or material resources to obtain such data. If the conditions are not allowed, it will lose its practical significance.

(6) Dynamic Principle. Evaluation of the community environment is a dynamic process. The selected index system must reflect the specific historical and current level of the research object and its development trend.

chart 1 Urban Reconstruction Community Environmental Impact Assessment System in Wenchuan Earthquake Stricken Areas

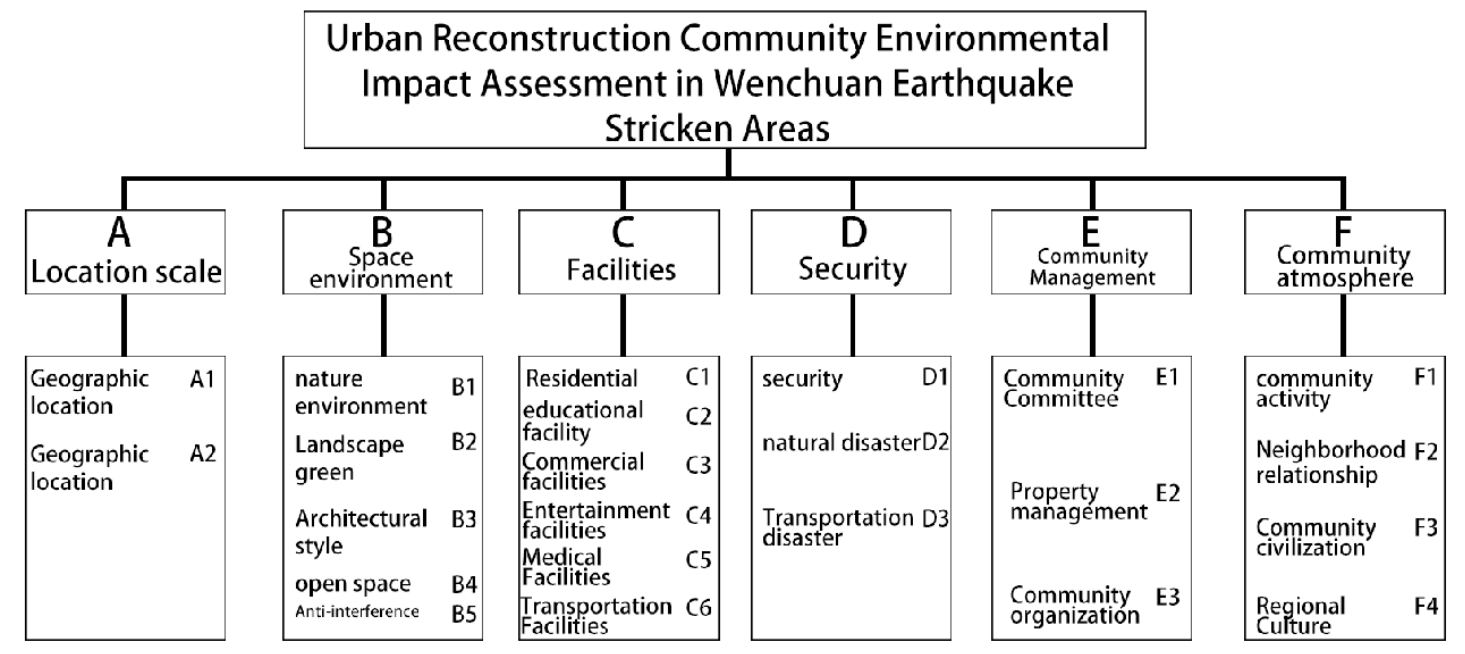

\section{Database Establishment}

Brief introduction of the project: Dujiangyan "One Neighborhood" is located in the northeastern part of Dujiangyan City. It is a permanent resettlement housing project for post-disaster urban and rural reconstruction supported Shanghai. It integrates living, education, medical care, sightseeing, 
shopping and leisure in one integrated community. It is a new pilot for exploring new modes of integrating the government, enterprises and social forces in urban renewal. To create a "harmonious home, living paradise, public park, ecological garden" for the planning concept,which make living functions as the main complex idea of the city Neighborhoods.The construction reflects Shanghai city design's concept and leve.

Using the method of questionnaire, obtain the first-hand information of the sample. Six firstlevel indicators, 27 second-level indicators were evaluated for the degree of importance in order to calculate the weight of the factors in the latter part of the year. The residents were evaluated on the satisfaction of 27 second-level indicators and combined with the latter part of the calculation to make a comprehensive assessment of the community and all aspects of the community. Questionnaire is divided into basic information, comprehensive evaluation, factor importance assessment and specific evaluation of four parts.

The measurement method of this research mainly adopts Likert scale, and uses the five-level system to qualitatively evaluate the index items. The quantitative standard is shown in Table 1.

Table 1 Five-level evaluation criteria

\begin{tabular}{c|c|c|c|c|c}
\hline & 5 & 4 & 3 & 2 & 1 \\
\hline \multirow{2}{*}{ Importance } & Very important & $\begin{array}{c}\text { Relatively } \\
\text { important }\end{array}$ & general & $\begin{array}{c}\text { Not very } \\
\text { important }\end{array}$ & $\begin{array}{c}\text { Very } \\
\text { unimportant }\end{array}$ \\
\hline \multirow{2}{*}{ satisfaction level } & Very satisfied & Quite satisfied & general & $\begin{array}{c}\text { Not very } \\
\text { satisfied }\end{array}$ & Very UNsatisfied \\
\cline { 2 - 6 } & Very Good & Quite well & general & Not good & Very bad \\
\hline
\end{tabular}

Level 1 factor, using the order of importance, from 1 to 6 , the more front ranking, the higher the degree of importance. While scoring, ranked first score is 6 , the second score is 5 , and so on, the sixth score of 1 .

A total of 80 questionnaires were sent out and 76 valid questionnaires were collected. Statistical analysis use software SPSS to establish the database.

\section{Indicator Weight}

\section{(1) Weight Calculation}

The study uses analytic hierarchy process to assign weights to indicators at all levels.. The Likert scale is calculated and converted into a 1-9 ratio scale method in AHP. According to the pairwise comparison method in AHP, a judgment matrix is obtained, and then the square root algorithm is used to calculate the eigenvector of the matrix - the relative weight [1]. According to the matrix theory, the eigenvector of the judgment matrix is the relative weight of the corresponding element to the upper element, so that the qualitative judgment of residents can be transformed into the quantitative description. In fact, as many scholars have pointed out[2][3][4][5], there are many flaws in 1-9 scale. One is that it has a thicker weighting order and is more different from people's judgment, and may appear contrary to the actual reverse order..The third is the consistency of the matrix and the thinking of the disjunction [6]. This article through the analytic hierarchy process softwareYAAHP0.5.2 converts the 1-9 scale type into $\mathrm{e}^{\wedge}(0 / 5) \sim \mathrm{e}^{\wedge}(8 / 5)$ scale for weight calculation. 
Table2 1-9 Grading comparison scale

\begin{tabular}{|c|c|c|}
\hline NO. & Importance level & \\
\hline 1 & Both indicators are equally important & 1 \\
\hline 2 & $\begin{array}{l}\text { The indicator } \alpha \mathrm{i} \text { is slightly more important than the } \\
\text { indicator } \alpha \mathrm{i}\end{array}$ & 2 \\
\hline 3 & $\begin{array}{l}\text { The index } \alpha \mathrm{i} \text { is significantly more important than } \\
\text { the index } \alpha \mathrm{i}\end{array}$ & 3 \\
\hline 4 & The index $\alpha \mathrm{i}$ is more important than the index $\alpha \mathrm{i}$ & 4 \\
\hline 5 & The index $\alpha \mathrm{i}$ is more important than the index $\alpha \mathrm{i}$ & 5 \\
\hline 6 & $\begin{array}{l}\text { The index } \alpha \mathrm{i} \text { is slightly less important than the index } \\
\alpha \mathrm{i}\end{array}$ & 6 \\
\hline 7 & $\begin{array}{l}\text { The index } \alpha \mathrm{i} \text { is significantly less important than the } \\
\text { index } \alpha \mathrm{i}\end{array}$ & 7 \\
\hline 8 & $\begin{array}{c}\text { The indicator } \alpha \mathrm{i} \text { is strongly unimportant than the } \\
\text { indicator } \alpha \mathrm{i}\end{array}$ & 8 \\
\hline 9 & The index $\alpha \mathrm{i}$ is less important than the index $\alpha \mathrm{i}$ & 9 \\
\hline
\end{tabular}

$\alpha \mathrm{ij}=\{2,4,6,8,1 / 2,1 / 4,1 / 6,1 / 8\}$ indicates that the importance level is between $\alpha \mathrm{ij}=\{1,3,5,7,1$ / $3,1 / 5,1 / 7,1 / 9\}$

Assignment between the corresponding values.

SPSS software was used to rebuild the community environmental level evaluation index score of Dujiangyan One Neighborhood. The order of importance of the first-level index is D>B $>C>E>$ A> F, and its assignment is shown in Table 3.

Table3 importance of the first-level index

\begin{tabular}{c|c|c|c|c|c|c}
\hline Indicator number & $\mathrm{A}$ & $\mathrm{B}$ & $\mathrm{C}$ & $\mathrm{D}$ & $\mathrm{E}$ & $\mathrm{F}$ \\
\hline $\begin{array}{c}\text { Importance assignment } \\
(\mathrm{hi})\end{array}$ & 2 & 5 & 4 & 6 & 3 & 1 \\
\hline
\end{tabular}

Depending on the score, assignments can be adjusted in the 1-9 range.

Assigne importance of the factors to the following formula for each pair of comparisons to obtain a pair of indicators of the two indicators.

$$
\alpha_{i j}=\left\{\begin{array}{cc}
h_{i}-h_{j}+1 & \left(h_{i}>h_{j}\right) \\
1 & \left(h_{i}=h_{j}\right) \\
1 /\left(h_{i}-h_{j}+1\right) & \left(h_{i}=h_{j}\right)
\end{array}\right.
$$

Several comparative indicators after $\frac{n(n-1)}{2}$ comparisons, determine the composition of the judgment matrix. 


$$
\mathrm{A}_{0}=\left[\begin{array}{cccccc}
1 & 1 / 4 & 1 / 3 & 1 / 5 & 1 / 2 & 2 \\
4 & 1 & 2 & 1 / 2 & 3 & 5 \\
3 & 1 / 2 & 1 & 1 / 3 & 2 & 4 \\
5 & 2 & 3 & 1 & 4 & 6 \\
2 & 1 / 3 & 1 / 2 & 1 / 4 & 1 & 3 \\
1 / 2 & 1 / 5 & 1 / 4 & 1 / 6 & 1 / 3 & 1
\end{array}\right]
$$

By YAAHP0.5.2 converted to $\mathrm{e}^{\wedge}(0 / 5) \sim \mathrm{e}^{\wedge}(8 / 5)$ scale, we get the matrix as follows.

$$
\mathrm{A}=\left[\begin{array}{llllll}
1.0000 & 0.5488 & 0.6703 & 0.4493 & 0.8187 & 1.2214 \\
1.8221 & 1.0000 & 1.2214 & 0.8187 & 1.4918 & 2.2255 \\
1.4918 & 0.8187 & 1.0000 & 0.6703 & 1.2214 & 1.8221 \\
2.2255 & 1.2214 & 1.4918 & 1.0000 & 1.8221 & 2.7183 \\
1.2214 & 0.6703 & 0.8187 & 0.5488 & 1.0000 & 1.4918 \\
0.8187 & 0.4493 & 0.5488 & 0.3679 & 0.6703 & 1.0000
\end{array}\right]
$$

Calculates the product $\mathrm{Mi}$ of each row of the matrix of the judgment matrix, and takes the calculated value int $\bar{W}_{i}$ e formula.

Calculate vector

Normalize vector $: \bar{W}$

$\mathrm{W}_{\mathrm{i}}=\frac{\bar{W}_{i}}{\sum_{i=1}^{n} \overline{W_{i}}}$

get $\mathrm{W}_{1}=0.1166, \quad \mathrm{~W}_{2}=0.2124, \quad \mathrm{~W}_{3}=0.1739, \quad \mathrm{~W}_{4}=0.2594, \quad \mathrm{~W}_{5}=0.1423, \quad \mathrm{~W}_{6}=0.0954$

Judgment matrix consistency index

After the above analysis and calculation, the weights of the level 1 indicators of Community $\mathrm{CI}=\frac{\lambda_{M A X}-n}{n-1}=\frac{6.000-6}{6-1}=0$

Environmental Assessment in Dujiangyan One Neighborhood are calculated to be $0.1166,0.2124$, $0.1739,0.2594,0.1423$ and 0.0954 .

According to this method, the index layer is calculated and the weight distribution is shown in Table 4.

\begin{tabular}{|c|c|c|c|c|c|c|c|c|c|}
\hline \multicolumn{5}{|l|}{ Guidelines layer } & \multicolumn{5}{|c|}{ Indicator layer } \\
\hline A Location scale & 0.1166 & A1 & 0.5498 & $\mathrm{~A} 2$ & 0.4502 & & & & \\
\hline $\begin{array}{l}\text { B Space } \\
\text { environment }\end{array}$ & 0.2124 & B1 & 0.1985 & B2 & 0.1985 & B3 & 0.1089 & B4 & 0.1331 \\
\hline & & B5 & 0.1985 & B6 & 0.1625 & & & & \\
\hline & & $\mathrm{C} 1$ & 0.1181 & $\mathrm{C} 2$ & 0.1443 & $\mathrm{C} 3$ & 0.0968 & $\mathrm{C} 4$ & 0.0648 \\
\hline C Facilities & 0.1739 & $\mathrm{C} 5$ & 0.1762 & C6 & 0.1762 & $\mathrm{C} 7$ & 0.1443 & $\mathrm{C} 8$ & 0.0793 \\
\hline $\begin{array}{l}\text { D Security } \\
\text { E Community }\end{array}$ & 0.2594 & D1 & 0.2866 & D2 & 0.2866 & D3 & 0.1921 & D4 & 0.2347 \\
\hline $\begin{array}{l}\text { Management } \\
\text { F Community } \\
\text { atmosphere }\end{array}$ & 0.1423 & E1 & 0.3289 & E2 & 0.4018 & E3 & 0.2693 & $\mathrm{~F} 4$ & 0.1807 \\
\hline
\end{tabular}

Table 4 Index Weight 
(2) statistical analysis of the importance of indicators

The order of importance of Dujiangyan One Neighborhood is ranked as: Security> Space Environment> Facilities Package> Community Management> Location Scale> Community Atmosphere. Showing great importance to safety and reflecting the people's safety awareness in disaster-stricken areas. The "community atmosphere" can not be taken seriously and reflected in post-disaster reconstruction. The emphasis on psychological reconstruction is not enough and needs to be strengthened.

The average scores of 27 important indicators of secondary indicators were calculated and presented in a more intuitive chart (Figure 2):

In "Location Size", the importance of "geography" is higher than "community size." In "space environment", "natural environment", "landscape greening" and "anti-interference"show high degree of attention, but the attention on "architectural style" is general.

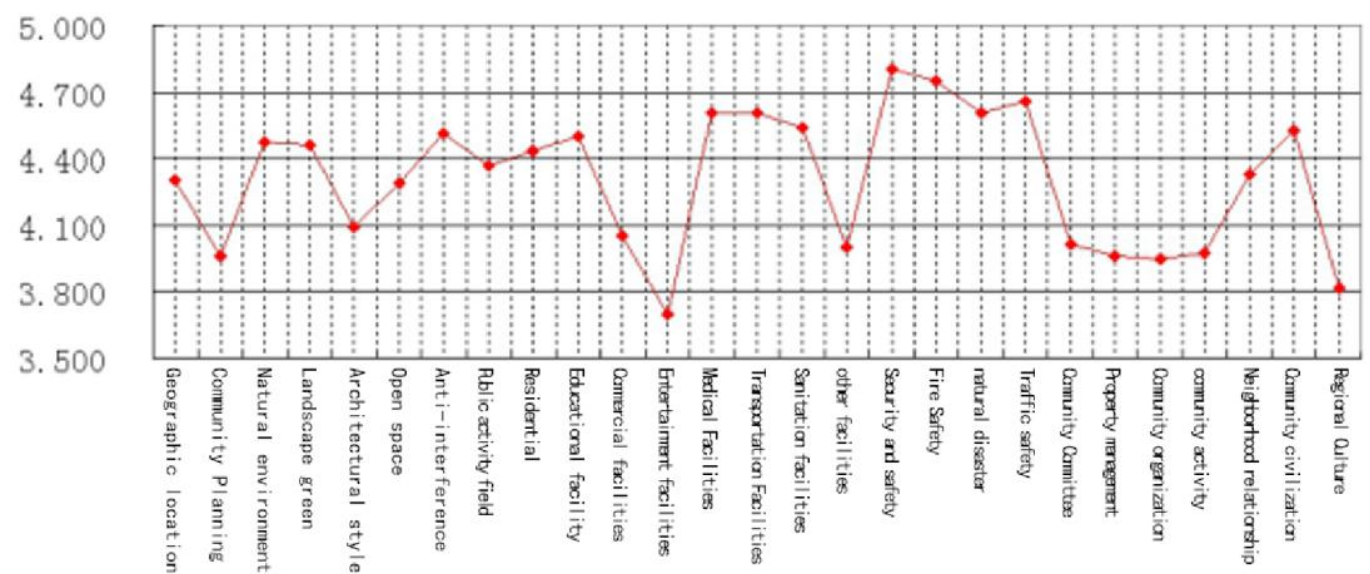

Chart 2 Metrics significance of the average statistics

"Facilities" such as "housing", "education facilities", "medical facilities", "transport facilities", "sanitation set "get more higher degree of importance, while entertainment facilities are emphasized.

In "Security", the safety factor has received the highest attention, of which "public security" scores the highest degree of importance.

The importance of "community management" factors is generally low. Among "community atmosphere", "neighborhood relationship" and "community

District civilization "is very important, the other two are still lower.

Visiblely, residents are concerned about hardware facilities, lack attention to software management such as management and community culture.

\section{Overview}

\section{(1) Evaluation Criteria}

Before Quantifing the comprehensive evaluation, we must first determine the quantitative evaluation criteria in order to assess the final evaluation results. In combination with the design of the Likert scale in the questionnaire design, we get the following quantitative criteria .

Table 5 Evaluation of quantitative standards

\begin{tabular}{c|c|c}
\hline Evaluation value $\mathrm{a}$ & Class \\
$\mathrm{a} \geq 4.5$ & very satisfied & M1 \\
$4.5>\mathrm{a} \geq 3.5$ & Quite satisfied & M2 \\
$3.5>\mathrm{a} \geq 2.5$ & general & M3 \\
$2.5>\mathrm{a} \geq 1.5$ & Not satisfied & M4 \\
$1.5>\mathrm{a}$ & Very dissatisfied & M5 \\
\hline
\end{tabular}


(2) Single Factor Evaluation

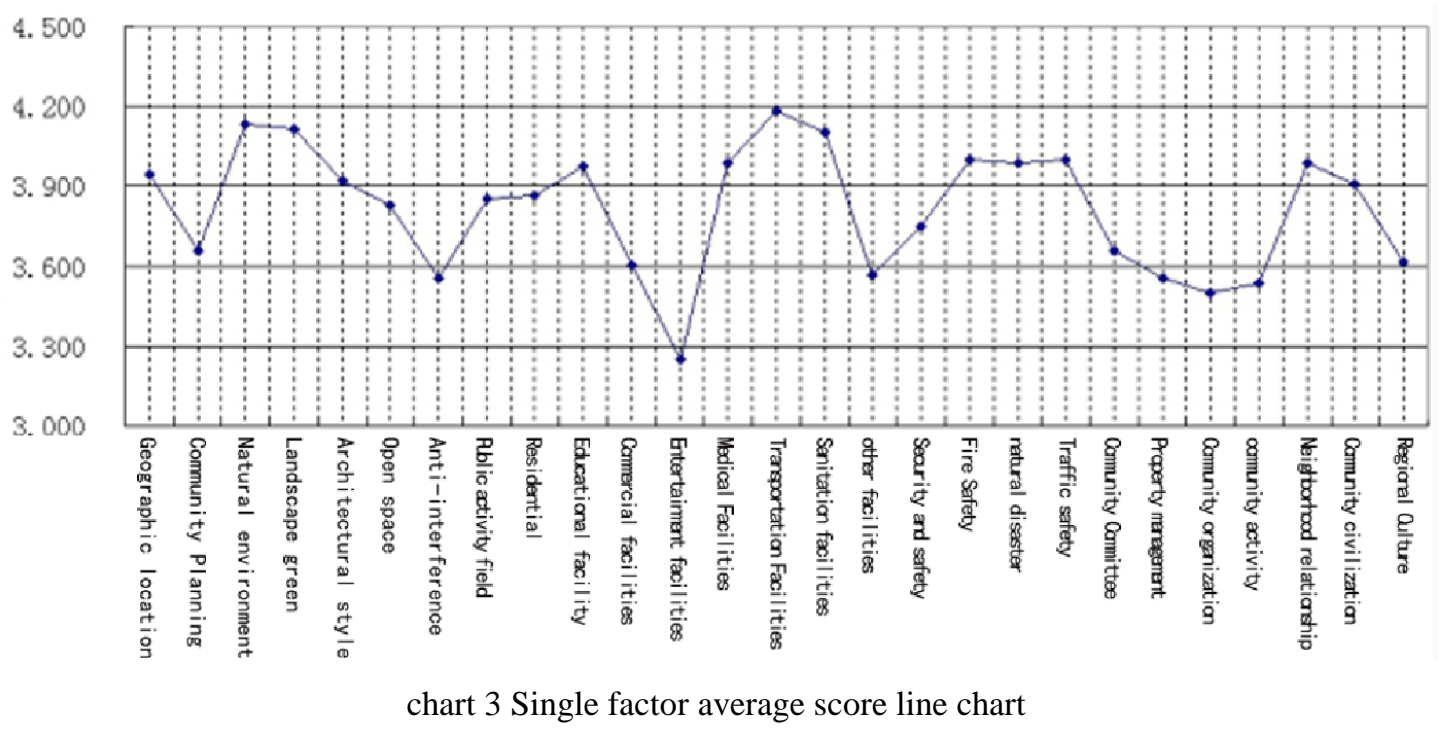

Fluctuation analysis from the fold line. Line fluctuation range smaller, in addition to entertainment facilities, satisfaction with an average score of 3.5 and above, indicating one neighborhood in the community environment in all aspects of the construction has made good results, while the relative coordination of various facilities, it is more conducive to Maximize the performance of various factors to achieve high satisfaction.

Satisfaction of the indicators compared to the peak trough, the peaks were the natural environment, education facilities, transport facilities, neighborhood relations and safety of the region; trough were community size, anti-interference, entertainment facilities, other facilities, community activities, Regional culture, and community management in this area.

Crest: (1) The historical and cultural city Dujiangyan, with its tourism as one of the leading industries, enjoys natural scenery with pleasant natural scenery. With the emphasis on restoration and reconstruction and the vigorous restoration and reconstruction of the ecological protection, it brings a good natural environment foundation to urban communities. (2) Reconstruction of public service facilities is also the guarantee for resuming normal life in the affected areas. Education and medical facilities are the key points of the disaster-stricken areas. Good achievements have also been made in the construction and operation of the disaster-stricken areas. (3) The first Neighborhood is close to Dujiangyan City and the infrastructure construction with high standards is close to urban areas . (4) The survey sample residents in the community are in accordance with the original administrative unit - the village, town unified arrangements. So the sample residents have a good neighborhood base. (5) Security and safety in Dujiangyan One Neighborhood needs to be improved.

Trough: (1) During the author's field visit, the biggest problem with the sample is the lack of popularity. This is also a major factor in the lower level of community In the case of a small population, large-scale and large-scale commercial facilities and recreational facilities lack popular support and are difficult to operate, making it difficult to scale commercial facilities and recreational facilities in the disaster areas Therefore, their satisfaction is low. (2) As the sample community layout pattern adopts the method of small group enclosure, the street density is large, which leads to the weak anti-interference ability of the community. (3) The overall trend of the three factors involved in community management is at a low level, which shows that while the hardware facilities environment has made great achievements, the software environment is lagging behind and needs to be strengthened. (4) Community culture helps to promote the sense of belonging and pride of communities. It needs to be strengthened in community culture construction.

\section{(3) Fuzzy Comprehensive Evaluation}

The environmental assessment of urban reconstruction in Wenchuan earthquake-stricken areas involves many aspects and many factors and is a comprehensive evaluation process. The author 
attempts to make a comprehensive quantitative evaluation of the evaluation objectives through fuzzy comprehensive Evaluation 1, combined with the database and index weights.

Fuzzy comprehensive evaluation steps:

(1) Establish six sets of factors $U=\{U A, U B, U C, U D, U E, U F\}=\{$ location size, space environment, facilities, safety and security, community management, community atmosphere\}, one for each factor set Fuzzy child

$\mathrm{U}_{\mathrm{A}}=\{\mathrm{A} 1, \mathrm{~A} 2\}, \mathrm{U}_{\mathrm{b}}=\{\mathrm{B} 1, \mathrm{~B} 2, \ldots \mathrm{B} 6\}, \ldots \mathrm{U}_{\mathrm{F}}=\{\mathrm{F} 1, \mathrm{~F} 2, \ldots \mathrm{F} 5\}$.

(2) Set up a set of weights.

Level 1 indicator layer weight: $\mathrm{A} 0=[0.117,0.212,0.174,0.259,0.142,0.096]$

Level 2 indicator layer weight: A0 $=[0.117,0.212,0.174,0.259,0.142,0.096]$

The basic idea of fuzzy comprehensive evaluation is to use the principle of fuzzy linear variate and the principle of maximum membership to consider all the factors related to the evaluated things and make a reasonable comprehensive evaluation. Fuzzy comprehensive decision-making evaluation is a very effective multi-factor decision-making method for making a comprehensive evaluation of things affected by many factors.

$\mathrm{A}_{\mathrm{A}}=[0.55,0.45]$

$\mathrm{A}_{\mathrm{B}}=\left[\begin{array}{llll}0.199,0.199,0.109, & 0.132,0.199,0.162\end{array}\right]$

$\mathrm{A}_{\mathrm{F}}=\left[\begin{array}{lll}0.221, & 0.27,0.328,0.181\end{array}\right]$

(3) Set up the evaluation set, $V=\{\mathrm{v} 1, \mathrm{v} 2, \mathrm{v} 3, \mathrm{v} 4, \mathrm{v} 5\}=\{$ bad, poor, fair, good, good $\}$.

(4) Through counting the SPSS database, we get the single factor fuzzy comprehensive evaluation matrix $R_{A}, R_{B} \ldots R_{F}$.

(5)Caculate each one-factor evaluation vector $\mathrm{W}_{\mathrm{A}}, \mathrm{W}_{\mathrm{B}} \ldots \mathrm{W}_{\mathrm{F}}$.

$\mathrm{W}_{\mathrm{A}}=\mathrm{R}_{\mathrm{A}} \cdot \mathrm{A}_{\mathrm{A}}=\left[\begin{array}{ll}0.55 & 0.45\end{array}\right]$

$\cdot\left[\begin{array}{lllll}0.329 & 0.382 & 0.210 & 0.066 & 0.013 \\ 0.211 & 0.382 & 0.289 & 0.092 & 0.026\end{array}\right]=\left[\begin{array}{lllll}0.276 & 0.382 & 0.246 & 0.078 & 0.019\end{array}\right]$

$\mathrm{W}_{\mathrm{B}}=\mathrm{R}_{\mathrm{B}} \cdot \mathrm{A}_{\mathrm{B}}=\left[\begin{array}{lllll}0.313 & 0.364 & 0.256 & 0.052 & 0.015\end{array}\right]$

$\mathrm{W}_{\mathrm{F}}=\mathrm{R}_{\mathrm{F}} \cdot \mathrm{A}_{\mathrm{F}}=\left[\begin{array}{lllll}0.285 & 0.3 & 0.355 & 0.048 & 0.012\end{array}\right]$

(6) Constructing the general objective fuzzy comprehensive evaluation matrix $\mathrm{R}_{0}$ from the single factor evaluation vector set, and calculating the total target comprehensive evaluation vector $\mathrm{W}_{0}$.

$\mathrm{W}_{0}=\mathrm{R}_{0} \cdot \mathrm{A}_{0}=\left[\begin{array}{ccccc}0.276 & 0.382 & 0.246 & 0.077 & 0.019 \\ 0.313 & 0.363 & 0.257 & 0.052 & 0.015 \\ 0.333 & 0.347 & 0.245 & 0.043 & 0.033 \\ 0.338 & 0.334 & 0.276 & 0.017 & 0.033 \\ 0.203 & 0.317 & 0.373 & 0.065 & 0.043 \\ 0.285 & 0.3 & 0.355 & 0.048 & 0.012\end{array}\right]$

$\cdot\left[\begin{array}{llllll}0.117 & 0.212 & 0.174 & 0.259 & 0.142 & 0.096\end{array}\right]=\left[\begin{array}{lllll}0.3 & 0.343 & 0.284 & 0.045 & 0.027\end{array}\right]$

(7) Fuzzy Comprehensive Evaluation Results

The fuzzy comprehensive evaluation result is obtained by multiplying the factor or the target vector by the evaluation set to obtain each comprehensive evaluation level.

Single Factor Comprehensive Rating:

$\mathrm{V}_{\mathrm{A}}=5 \mathrm{X} 0.276+4 \mathrm{X} 0.382+3 \mathrm{X} 0.246+2 \mathrm{X} 0.077+1 \mathrm{X} 0.019=3.819$

$\mathrm{V}_{\mathrm{B}}=3.907, \mathrm{~V}_{\mathrm{C}}==3.907, \mathrm{~V}_{\mathrm{D}}=3.921, \mathrm{~V}_{\mathrm{E}}=3.575, \mathrm{~V}_{\mathrm{F}}=3.798$

Overall Objective Comprehensive rating: 
$\mathrm{V}_{0}=5 \mathrm{X} 0.300+4 \mathrm{X} 0.343+3 \mathrm{X} 0.284+2 \mathrm{X} 0.045+1 \mathrm{X} 0.027=3.841$

Dujiangyan one Neighborhood overall evaluation score is 3.841 , with reference to the evaluation of quantitative standards, the evaluation rating is Quite Good. In the guidelines layer, the spatial environment, facilities and facilities, safety and security score are higher than 3.9, and community management and community culture score are relatively low.

\section{Conclusion}

The results of high standards of post-disaster reconstruction achieved and the effect is very significant for the residents of disaster areas to provide a good student.

Live to rebuild the foundation, but there are many problems in the construction of community soft environment. In order to achieve social harmony and sustainable development in the disaster areas, there is still a long way to go in community building. From feedback, the disaster area community environment construction needs to strengthen community management, community culture, focusing on facilities management operations.From many aspects to improve residents' sense of belonging and pride of the community to promote the reconstruction of disaster areas living condition.So that we can make the people in disaster area to live a well-being life.

\section{References}

[1]Chen Xiuwen.Discussion on Evaluation Index System of Public Service Facilities in Urban Residential Community[D].ChongQing University, (2007)

[2]Shu Kang, Liang Zhenhan.Systems Engineering Theory and Practice, (1990)

[3]Hou Yueheng, Shen Dejia.Index Scale and Comparison with Several Scales [J] Systems Engineering Theory and Practice(1995) p.43-46

[4]Yang Yongqing, $\mathrm{Xu}$ Xianyun.Improved Analytic Hierarchy Process for Comprehensive Evaluation of Mine Safety Management [J] Systems Engineering(1999) p.121-125

[5]Chen Qian, Wang Shenchen.System Engineering(1996) p.18-21

[6]Lv Yuejin, Zhang Wei.Exponential scaling in AHP scale system important role [J] Journal of Systems Engineering(2003) p.453-456 